\title{
Assessment of Barriers in Subject Recruitment for Clinical Trials
}

\author{
Ruckmani A*, Vishaly S, ArunKumar R, Lakshmipathy Prabhu R and Priya A
}

Department of Pharmacology, Chettinad Hospitals and Research Institute, Kelambakkam, Chennai, India

\begin{abstract}
Aminoglycosides are very effective against most Gram negative infections, but nephrotoxicity and electrolyte imbalance caused by these compounds pose problems in patients where electrolyte balance needs to be watched closely. The electrolyte imbalance contributes to derangement of the renal functions. This issue has been addressed by changing the dosing schedule and supplementing the patients with these electrolytes. However electrolyte administration and adjusting aminoglycoside doses have their own demerits. In our present study we investigated the effects of Pyridoxal phosphate on electrolyte balance when given in combination with aminoglycosides. Our findings suggest that Pyridoxal phosphate prevents aminoglycoside induced electrolyte imbalance, a finding which has not been reported previously. These results should be considered as an important input regarding prevention of aminoglycoside induced decrease in electrolyte levels. Further studies will help healthcare providers to manage patients more efficiently, in whom electrolytes balance may need to be observed.
\end{abstract}

\section{Introduction}

Clinical trials are conducted across many countries and the success of a clinical trial will depend on many factors such as commitment of the sponsor, competency of the investigator, appropriate study design \& statistical analysis, adequate resources and availability of trial subjects.

Recruitment of the subjects and retention of the enrolled subjects till completion of the trial will certainly decide the success of clinical trials. Low subject recruitment will prolong the trial duration and can have various negative implications. The sponsor would find it difficult to meet timelines of regulatory submission and bear additional expenditure of prolonged trial. Inability to meet recruitment targets may cause premature closure of study sites and when it occurs at multiple sites, it may lead to termination of the trial. If enrollment of required number of subjects fails, it may affect the statistical power of the trial and validity of the data generated. Low subject recruitment will also affect the interest of the study team including the investigator, CRA, monitor and sponsor [1-4]. Therefore poor subject recruitment is likely to impede successful evaluation of new drug interventions and adversely influence clinical trial programs [5].

Hence it was planned to conduct a survey to evaluate the impact of different barriers in subject recruitment among investigators. The study was carried out by the Division of Clinical Research and Experimental Medicine, Department of Pharmacology, Chettinad Hospitals and Research Institute, Chennai, India.

\section{Methods}

The study was approved by the Institutional Ethics committee, Chettinad Hospitals and Research Institute, Chennai.

The survey was conducted among investigators involved in clinical trials. The investigators were selected from the information available at the time of the study in Clinical Trials Registry - India (CTRI) (www.ctri. nic.in) and by contacting the Indian Clinical Research Organizations (CROs). They were all principal investigators participating in different national and international clinical trials.

The tool to assess the barriers was designed in the form of a questionnaire consisting of 25 potential barriers as given in table 1. Invitations were sent to the investigators via email, seeking their consent to take part in the survey. The questionnaire was then sent to willing participants. They were requested to grade each of the barriers as mild, moderate, severe or not a barrier based on their experience and personal judgment. Each barrier has been concluded to be mild, moderate, severe or not a barrier, depending on the maximum number of responses received for a grade.

\section{Results}

Invitations were sent to 1004 investigators and among them 105 accepted the invitations. They were sent subsequently with the questionnaire designed to assess the severity of recruitment barriers. The response rate was found to be $10.45 \%$.

The responses were compiled, analyzed. Based on the maximum number of investigators grading a barrier into any one of the four categories (mild, moderate, severe or not a barrier), a specific barrier has been concluded as mild, moderate, severe or not a barrier. The responses are graphically represented in figures 1,2 and 3 .

12 barriers were identified as mild and 11 moderate barriers. 2 barriers were rated severe barriers.

"Negative impact from the media" and "rare disease" was rated severe barriers by $34 \%$ and $50 \%$ of respondents respectively.

"Complex nature of the study trial design", "Eligibility criteria is too specific", "Stay in the hospital", "The quantity and frequency of blood collected for analysis threaten the subjects", "Lack of awareness about the clinical trial", "Less patient recruitment period given to investigators", "Selecting a site in inappropriate epidemiological area", "Large sample size", "Inconvenient schedule of clinic visits for subjects", "Distance between site and residence of the subject" and "Fear of taking a new drug" were rated as moderate barriers.

Corresponding author: Ruckmani A, Professor and Head of the Department of Pharmacology, Chettinad Hospitals and Research Institute, Kelambakkam, Chennai, India, Tel: 9144 47429211; Fax: 9144 47413300; E-mail: ruckmani.nirmal@gmail.com

Received November 24, 2011; Accepted January 14, 2012; Published January 20, 2012

Citation: Ruckmani A, Vishaly S, ArunKumar R, Lakshmipathy Prabhu R, Priya A (2012) Assessment of Barriers in Subject Recruitment for Clinical Trials. J Clinic Res Bioeth 3:125. doi:10.4172/2155-9627.1000125

Copyright: (c) 2012 Ruckmani A, et al. This is an open-access article distributed under the terms of the Creative Commons Attribution License, which permits unrestricted use, distribution, and reproduction in any medium, provided the original author and source are credited. 


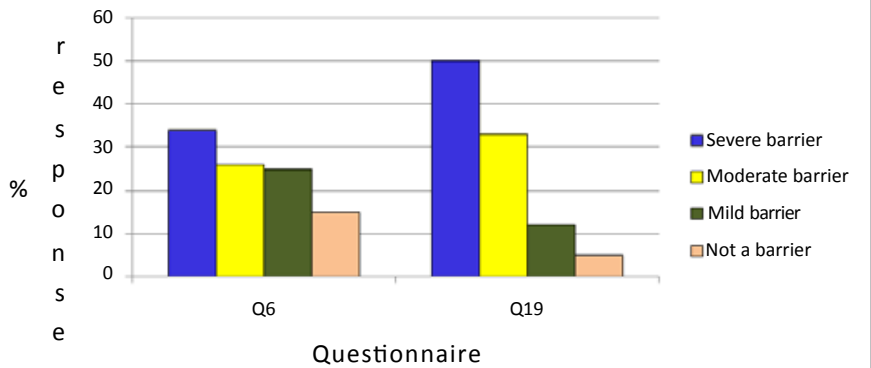

Figure 1: Percentage Responses to Severe barriers.

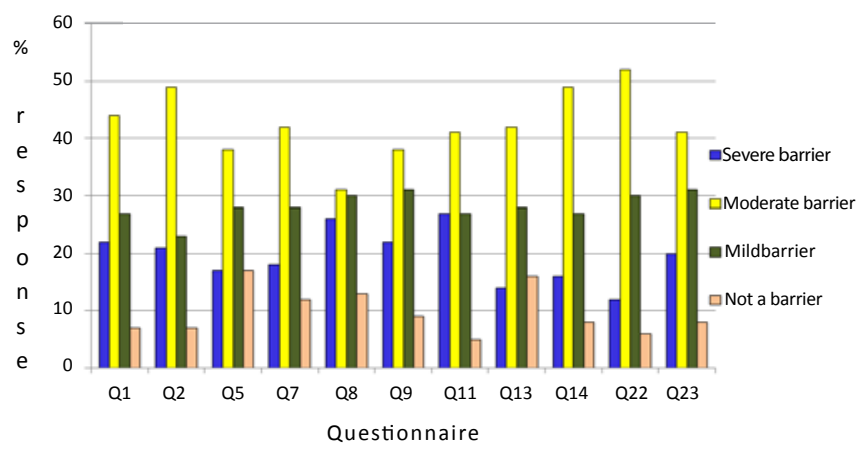

Figure 2: Percentage Responses to Moderate barriers.

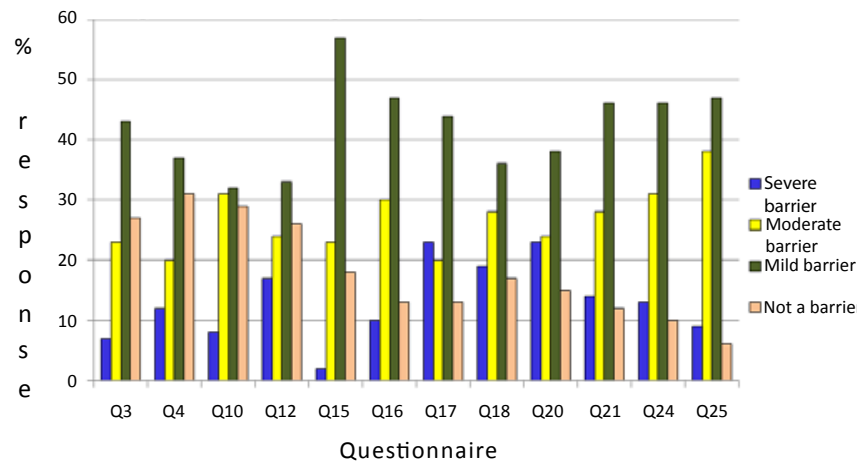

Figure 3: Percentage Responses to Mild barriers.

The remaining 12 barriers (questions- $3,4,10,12,15,16,17,18$, $20,21,24$ and 25 of table 1) were categorized as mild barriers. "Local cultural practices" was rated by $57 \%$ of respondents as mild barrier.

Though each barrier was graded as "not a barrier" by few of the respondents, based on the maximum scores obtained for a specific barrier, none of the barriers could be categorized as "not a barrier".

\section{Discussion}

The response rate for accepting the invitation was found to be low (10.45\%) compared to $69 \%$ response rate reported by Anne Spaar et al. [6] in 2009 in a similar study. However the study done by Anne Spaar et al. [6] was a postal survey and the present study was done by email communication. The reason for the low response rate among the participants may be due to a) this study being the first of its kind; the investigators would not have been willing to participate in the study, b) time constraints of the investigators [6].
As per Anne Spaar et al. [6] "time constraints" followed by "difficulties in including identified eligible patients" was found to have the most negative impact on subject recruitment [6]. In this survey, "negative impact from the media" and "trial conducted for a rare disease" was rated severe barriers for subject recruitment.

This study reveals that the investigators are concerned about the negative views expressed by the media about clinical trials. There could be a few instances where media exaggerated drug related issues. At the same time some of the infamous trials conducted were actually brought to light by the media [7-9]. The media information will make the investigators and general public aware of clinical trials and ethics. However, some times the negative information provided by the media may adversely affect the opinion and attitude of the public towards clinical research and thereby their participation in clinical trials, especially in the areas where the exposure to media is significant. The prospective investigators may not actively come forward to help conduct clinical trials fearing adverse publicity and it will affect clinical development of new drug candidates. The media can play a significant role by providing information on the importance of conducting clinical trials, how they contribute to new drug introduction, how the subjects play a crucial role in this mission of new drug development by subjecting themselves to the clinical trials. At the same time, the media need not withhold any adverse information about the clinical trial as this would also help both the public and key players for preventing such occurrences of adverse events in future.

The other barrier rated severe is "rare disease" and it is understandable that investigators may find it difficult to recruit when the incidence or prevalence of the target disease is low. This observation is important especially in the current clinical trial scenario where orphan drug development is being emphasized and encouraged. To address this barrier, a collective approach should be made to create and maintain the data bases of rare disorders and facilitate clinical trials in this domain.

$52 \%$ of respondents rated "Distance between site and residence of the subject" as a moderate barrier. The travel time to reach the trial site may be a limiting factor for subjects to take part in the trial and this will also affect the scheduled follow up visits. This could be overcome by selecting subjects who reside closer to the trial sites, providing adequate compensation for travel expenses and expanding the network of clinical trial sites to suburban and rural areas so that the travel distance is reduced.

$49 \%$ have rated "Eligibility criteria for subject recruitment are too specific" and "Inconvenient schedule of clinic visits for subjects" as moderate barriers. These barriers can be addressed by a well designed study protocol and procedures taking into consideration the practical applicability of eligibility criteria and inconveniences that the study procedures may have on the subject's day-to-day life. The number of visits in a clinical trial should be balanced between the "maximum number of visits that could be possible for the subjects without affecting their routine work" and "minimum number of visits required to evaluate the trial end points without compromising the outcome". It can probably be fixed after getting feedback from the investigators and potential study subjects.

Though none of the barriers qualified for the category "Not a barrier" based on the analysis of data, more than $25 \%$ of respondents have considered the following four barriers as not a barrier. They are "Participants feel the compensation given is low", "Many trials taking place in same area in the same period", "Stipulated interval of three 
1. Complex nature of the study trial design

2. Eligibility criteria for subject recruitment is too specific

3. Due to randomization there is no confirmation for access to the new treatment for participants

4. Participant feel the compensation given is low

5. Stay in the hospital

6. Negative impact from the media

7. The quantity and frequency of blood collected for analysis threaten the subjects

8. Lack of awareness about the clinical trial

9. Less patient recruitment period given to investigators

10. Stipulated interval of three months for participation in various trials

11. Selecting a site in, inappropriate epidemiological area

12. Many trials taking place in same area in the same period

13. Large sample size

14. Inconvenient schedule of clinic visits for subjects

15. Local cultural practices (fasting on Friday, full moon day, festival restrictions for travel etc)

16. Education of subject and his family members

17. Migration of subject after screening

18. People conducting trial being unaware of the local language (in rural and in tribal areas)

19. In case of rare disease

20. Female subject becoming pregnant between screening and recruitment

21. Family events (death, illness of family members)

22. Distance between site and residence of the subject

23. Fear of taking a new drug

24. Sudden withdrawal of subject after screening (for personnel reasons)

25. Inter current illness after screening

Table 1: Questionnaire to assess barriers in subject recruitment for clinical trials

months for participation in various trials" and "Due to randomization there is no confirmation for access to the new treatment for participants".

"Female subject becoming pregnant between screening and recruitment" has been considered as mild barrier by $38 \%$ of respondents, moderate by $24 \%$, severe by $23 \%$ and not a barrier by $15 \%$ of respondents. This barrier can be overcome by educating and convincing the potential participants the importance of postponing pregnancy. The investigators can avoid female subjects for whom it may be difficult to avoid pregnancy such as recently married women and women who have not completed their family life. Female subjects getting pregnant during the trial will add more burden to the sponsor and investigator as there are long term reporting and monitoring responsibilities [10].

Jean Sullivan has classified the barriers into 1) subject-related barriers, 2) investigator-related barriers, 3) protocol-related barriers, and 4) "other" barriers [11].

The protocol related barriers can be avoided or minimized if the details such as number of subjects required, eligibility criteria for trial participation and study procedures are finalized based on the dedicated field research on study feasibility. It has to be emphasized that recruitment is a shared responsibility of sponsor and site personnel. The recruitment targets should be validated by the sponsor before the sites are assigned and preferably by a meticulous feasibility assessment plan [12].

Coordinated effort from the sponsor and site personnel will be required to overcome the subject related barriers. It can be said that the entire outcome of the clinical trial and probably the new drug development process will mostly depend on successful subject recruitment. Hence adequate resources in terms of staff and infrastructure have to be allocated to reduce the intensity of subject related barriers. The potential subjects have to be educated and motivated to participate in the trials without unduly influencing them in the due process.
The barriers related to the investigators can be reduced by doing selection of sites and investigators as per well defined \& evaluable criteria specified in the respective standard operating procedures (SOPs). The selection procedure should be such that it has to identify the experienced investigators who can foresee the barriers in their site and evolve a system to overcome the barriers. The activities to overcome the barriers may include education, motivation, training and making the investigators aware of the nuances of clinical trials and regulatory guidelines.

Though media impact is rated severe barrier in this survey, it has to be accepted that media such as news papers, magazines and internet can all aid in faster patient recruitment also. An effective strategy has to be framed on utilization of media resources to enhance the recruitment rates. The strategy may include steps to educate media personnel in the domain of clinical trials, regulatory requirements and ethics. Advertisements can be effectively used to attract the potential participants to come forward to take part in the trials without compromising the ethical issues.

\section{Conclusion}

The present survey was conducted among 105 investigators to assess the severity of various barriers in subject recruitment. Two barriers namely, "negative impact from the media" and "trial conducted in a rare disease" are concluded as severe barriers by the participants. 11 are designated moderate barriers and 12 as mild. None of the barriers is categorized as "Not a barrier'. Such rating of the barriers can help sponsors and investigators to foresee these barriers well in advance and evolve strategies to overcome them.

\section{Acknowledgement}

The Authors would like to thank all the investigators for their valuable contribution to the study.

\section{References}

1. Ross S, Grant A, Counsell C, Gillespie W, Russell I, et al. (1999) Barriers to participation in randomised controlled trials: a systematic review. J Clin Epidemiol 52: 1143-1156.

2. Hunninghake DB, Darby CA, Probstfield JL (1987) Recruitment experience in clinical trials: literature summary and annotated bibliography. Control Clin Trials 8: 6 S-30S.

3. Lovato LC, Hill K, Hertert S, Hunninghake DB, Probstfield JL (1997) Recruitment for controlled clinical trials: literature summary and annotated bibliography. Control Clin Trials 18: 328-352.

4. Genevieve Frank (2004) Current challenges in clinical trial patient recruitment and enrollment. SoCRA 30-37.

5. Spilker B, Cramer JA (1992) Patient Recruitment in Clinical Trials. Raven Press New York 365 pages.

6. Spaar A, Frey M, Turk A, Karrer W, Puhan MA (2009) Recruitment barriers in a randomized controlled trial from the physicians' perspective: a postal survey. BMC Med Res Methodol 9:14.

7. Francis Weyzig, Irene Schipper (2008) SOMO briefing paper on ethics in clinical trials. Examples of unethical trials 1-16.

8. Moynihan R, Bero L, Ross-Degnan D, Henry D, Lee K, et al. (2000) Coverage by the news media of the benefits and risks of medications. $N$ Engl $\mathrm{J} \mathrm{Med}$ $342: 1645-1650$

9. Schwitzer G (2008) How do US journalists cover treatments, tests, products, and procedures? An evaluation of 500 stories. PLoS Med 5: e95.

10. Deborah Covington, Laura McKain, MD (2011) Monitoring Drug Exposure in Pregnancy. Applied Clinical Trials Online.

11. Jean Sullivan (2004) Subject Recruitment and Retention: Barriers to Success, Study findings identify various factors affecting subject enrollment in today's clinical trial world. Applied Clinical Trials Online.

12. Lars-Olof Eriksson, Beth D Harper (2002) Successful Subject Recruitment: One Size Does Not Fit All. Applied Clinical Trials Online. 